\title{
Determination of flexibility factors in curved pipes with end restraints using a semi-analytic formulation
}

\author{
E.M.M. Fonseca ${ }^{a, *}$, F.J.M.Q. de Melo ${ }^{b}$, C.A.M. Oliveira ${ }^{b}$ \\ a Department of Applied Mechanics, Polytechnic Institute of Bragança, Campus De Sta Apolonia Apartado 134, Bragança 5301 857, Portugal \\ ${ }^{\mathrm{b}}$ Department of Mechanical Engineering and Industrial Management, Faculty of Engineering, University of Porto, Portugal
}

Received 17 January 2002; revised 30 August 2002; accepted 17 September 2002

\begin{abstract}
Piping systems are structural sets used in the chemical industry, conventional or nuclear power plants and fluid transport in generalpurpose process equipment. They include curved elements built as parts of toroidal thin-walled structures. The mechanical behaviour of such structural assemblies is of leading importance for satisfactory performance and safety standards of the installations. This paper presents a semi-analytic formulation based on Fourier trigonometric series for solving the pure bending problem in curved pipes. A pipe element is considered as a part of a toroidal shell. A displacement formulation pipe element was developed with Fourier series. The solution of this problem is solved from a system of differential equations using mathematical software. To build-up the solution, a simple but efficient deformation model, from a semi-membrane behaviour, was followed here, given the geometry and thin shell assumption. The flexibility factors are compared with the ASME code for some elbow dimensions adopted from ISO 1127. The stress field distribution was also calculated. (C) 2002 Published by Elsevier Science Ltd.
\end{abstract}

Keywords: Curved pipe; Pure bending; Flexibility factor; Semi-analytic formulation; Fourier series

\section{Introduction}

Pipe bends play a very important role in the global piping arrangement, as they not only allow flow direction change, but they also absorb thermal expansions and longitudinal deformations from adjacent tangent parts or other straight pipe elements [1]. Computer codes have been used extensively to expedite calculations of deflections, reactions and stresses both in piping system and pressure vessels. In order to determine such calculations, it is important to know flexibility factor values, where the case of pure bending is one of the most used in design [2]. Many experiments and theories have been presented to demonstrate and explain the flexibility and stresses of a curved pipe compared with elastic beam theory. In this paper we will present the results of flexibility factors using a formulation for a curved pipe subjected to uniform bending moment. We have determined the flexibility factor when the curved pipe was fitted with

\footnotetext{
* Corresponding author. Tel.: +351-273-303-157; fax: +351-273-313051 .

E-mail address: efonseca@ipb.pt (E.M.M. Fonseca).
}

thin or thick flanges. Throughout this formulation beam theory combined with the shell membrane equations is assumed.

\section{Equation formulations}

\subsection{Essential assumptions}

The deformation field refers to membrane strains and curvature variations.

The following assumptions [3] were considered in the present analysis:

(a) the curvature radius $R$ is assumed much larger than the section radius $r$;

(b) a semi-membrane deformation model is adopted and neglects the bending stiffness along the longitudinal direction of the toroidal shell but considers the meridional bending resulting from ovalization;

(c) the shell is thin and inextensible along the meridional direction. 


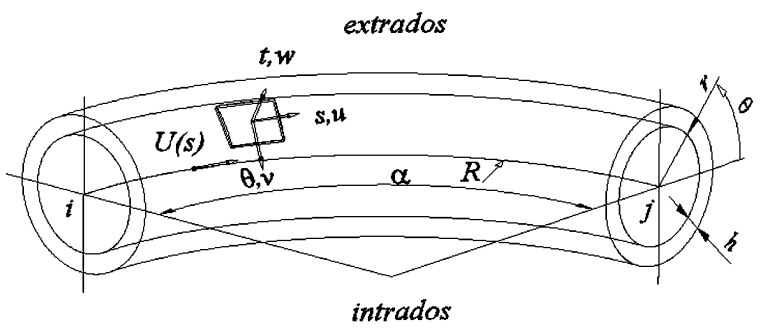

Fig. 1. Geometric parameters for the curved pipe.

Fig. 1 shows the essential parameters defining the pipe bend geometry.

\subsection{The displacement field}

For the displacement field we have superposed the effect of the rotation with trigonometric terms, for problems by symmetric bending

$\varphi(x)=\frac{x}{L} \delta \alpha+\sum_{k \geq 1}^{n x} r_{k} \sin \frac{k \pi x}{L}$ (complete set of terms)

$\varphi(x)=\frac{x}{L} \delta \alpha+\sum_{k \geq 1}^{n x} r_{k} \frac{L}{(2 k-1) \pi}$

$$
\times \sin \frac{(2 k-1) \pi x}{L} \quad \text { (with odd terms) }
$$

The displacement field for the curved beam coincident with a central arch is:

$W(x)=-\int \varphi(x) \mathrm{d} x$

$U(x)=-\frac{1}{R} \int W(x) \mathrm{d} x$

Here, trigonometric terms for section ovalization are included

$$
\sum_{k \geq 1}^{n x} \cos \frac{(2 k-1) \pi x}{2 L}
$$$$
\times \sum_{i \geq 2}^{n \theta}-\frac{a_{k i}}{i} \sin i \theta \quad \text { (for } v \text { displacement) }
$$

$$
\sum_{k \geq 1}^{n x} \cos \frac{(2 k-1) \pi x}{2 L}
$$

$$
\times \sum_{i \geq 2}^{n \theta} a_{k i} \cos i \theta \quad \text { (for } w \text { displacement) }
$$

Similar expressions are defined for the transverse section warping displacement field. Thus, expressions for warping $u$-displacement in transverse sections of the curved pipe either with rigid or thin flanges are:

$\sum_{k=1}^{n x} \sin \frac{k \pi x}{L} \sum_{i \geq 2}^{n \theta} b_{k i} \cos i \theta \quad$ (for rigid flanges) $\sum_{k=1}^{n x} \sin \frac{k \pi x}{2 L} \sum_{i \geq 2}^{n \theta} b_{k i} \cos i \theta \quad$ (for thin flanges)

(6b)

Finally, following the formulation proposed by Thomson [6], the displacement field in a curved pipe resulting from the superposition of displacement shell and the complete Fourier expansion for ovalization and warping terms, is:

$u=U(x)+(r \cos \theta) \varphi(x)+\sum_{k=1}^{n x} \sin \frac{k \pi x}{L}$

$\times \sum_{i \geq 2}^{n \theta} b_{k i} \cos i \theta, \quad$ (for rigid flanges)

$u=U(x)+(r \cos \theta) \varphi(x)+\sum_{k=1}^{n x} \sin \frac{k \pi x}{2 L}$

$\times \sum_{i \geq 2}^{n \theta} b_{k i} \cos i \theta, \quad$ (for thin flanges)

$v=-W(x) \sin \theta+\sum_{k \geq 1}^{n x} \sum_{i \geq 2}^{n \theta}-\frac{a_{k i}}{i}\left(\frac{\cos (2 k-1) \pi x}{2 L}\right)(\sin i \theta)$

$w=+W(x) \cos \theta+\sum_{k \geq 1}^{n x} \sum_{i \geq 2}^{n \theta} a_{k i}\left(\cos \frac{(2 k-1) \pi x}{2 L}\right)(\cos i \theta)$

Depicting the displacement field in a condensed matrix representation

$\left\{\begin{array}{c}u \\ v \\ w\end{array}\right\}=\left[\begin{array}{ccc}\frac{x^{3}}{6 L R}+r \cos \theta \frac{x}{L} & 0 & \left\{C S_{i}\right\} \\ \frac{x^{2}}{2 L} \sin \theta & \left\{S_{i}\right\} & 0 \\ -\frac{x^{2}}{2 L} \cos \theta & \left\{C S_{i}\right\} & 0\end{array}\right]\left\{\begin{array}{c}\delta \alpha \\ \underset{\sim}{r} \\ \underset{\sim}{a} \\ \underset{\sim}{b}\end{array}\right\}$

where

$$
\begin{aligned}
& \left\{C S_{i}\right\}=\sum_{k=1}^{n x} \sin \frac{k \pi x}{L} \sum_{i \geq 2}^{n \theta} b_{k i} \cos i \theta \text { or } \\
& \left\{C S_{i}\right\}=\sum_{k=1}^{n x} \sin \frac{k \pi x}{2 L} \sum_{i \geq 2}^{n \theta} b_{k i} \cos i \theta \\
& \left\{S_{i}\right\}=\sum_{k \geq 1}^{n x} \sum_{i \geq 2}^{n \theta}-\frac{a_{k i}}{i}\left(\frac{\cos (2 k-1) \pi x}{2 L}\right)(\sin i \theta) \text { and } \\
& \left\{C S_{i}\right\}=\sum_{k \geq 1}^{n x} \sum_{i \geq 2}^{n \theta} a_{k i}\left(\cos \frac{(2 k-1) \pi x}{2 L}\right)(\cos i \theta) .
\end{aligned}
$$

\section{1}

182

183

184

185

186

187

188

189

\subsection{The deformation field} considers that the pipe undergoes a semi-membrane strain 223 field. The strain field is given by the following equations

69

174

\section{5} .

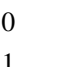

(

s.
, 
used by Melo, Flügge and Kitching [3-5]

$$
\begin{aligned}
\underset{\varepsilon}{\varepsilon} & =\left\{\begin{array}{c}
\varepsilon_{x} \\
\gamma_{x \theta} \\
K_{\theta}
\end{array}\right\} \\
& =\left[\begin{array}{ccc}
\frac{\partial}{\partial x} & -\frac{\sin \theta}{R} & \frac{\cos \theta}{R} \\
\frac{1}{r} \frac{\partial}{\partial \theta}+\frac{\sin \theta}{R} & \frac{\partial}{\partial x} & 0 \\
0 & -\frac{1}{r^{2}} \frac{\partial}{\partial \theta} & \frac{1}{r^{2}} \frac{\partial^{2}}{\partial \theta^{2}}
\end{array}\right]\left\{\begin{array}{l}
u \\
v \\
w
\end{array}\right\}
\end{aligned}
$$

where $\varepsilon_{x}$ is the longitudinal membrane strain, $\gamma_{x \theta}$ is the shear strain and $K_{\theta}$ is the meridional curvature from ovalization.

The element stiffness matrix $K$ is calculated from the matrix equation:

$[K]=\int_{x=0}^{x=L} \int_{\theta=0}^{\theta=2 \pi}[B]^{\mathrm{T}}[D][B] r \mathrm{~d} x \mathrm{~d} \theta$

The integration of this equation is extended to the pipe surface, where matrix $[B]$ results from:

$[B]=[L] \times[N]$

Given that the pipe is inextensible along the meridional direction, no contribution for elastic strain energy arises from such strains. The elasticity matrix $D$ appears with a simpler algebraic definition, having deleted the contribution of off-diagonal terms with a Poisson factor:

$D=\left[\begin{array}{ccc}\frac{E h}{1-\nu^{2}} & 0 & 0 \\ 0 & \frac{E h}{2(1+\nu)} & 0 \\ 0 & 0 & \frac{E h^{3}}{12\left(1-\nu^{2}\right)}\end{array}\right]$

where $E$ is the elasticity modulus, $h$ is the pipe thickness and $\nu$ is Poisson's is ratio.

The stress fields are determined by:

$\sigma=\left\{\begin{array}{c}N_{x x} \\ N_{x \theta} \\ M_{\theta \theta}\end{array}\right\}=[D]\left\{\begin{array}{c}\varepsilon_{x x} \\ \gamma_{x \theta} \\ K_{\theta \theta}\end{array}\right\}$

The matrix force-displacement equation for the curved pipe element is

$[K] \underset{\sim}{\delta_{\mathrm{e}}}=\underset{\sim}{F}$

where $K$ is the stiffness matrix and $\delta_{\mathrm{e}}$ is a nodal unknown displacement vector.

\section{Determination of flexibility factor in curved pipes} subjected to uniform bending in the curvature plane

The flexibility coefficient is a parameter that makes possible an accurate calculation for reactions at restraints and attachments in a piping system. For that task, one can use design codes either based on displacement or force methods, having included the flexibility factor as a stiffness reduction parameter for beam elements, whenever curved pipes are involved. In high temperature loading, creep phenomena can be present and a flexibility factor may be used to evaluate the time-dependent variation of the curved pipe bending resistance. The flexibility coefficient is calculated as follows

$K=\frac{M_{\mathrm{C}}(\delta \alpha)}{M_{\mathrm{S}}(\delta \alpha)}$

where $M_{\mathrm{C}}(\delta \alpha)$ is a resistant moment in a curved pipe subjected to a bending angle $\delta \alpha$ at the edge and $M_{\mathrm{S}}(\delta \alpha)$ is the corresponding bending moment in a straight pipe, with a length equal to that of the curved one and subjected to the same bending angle at the edge.

The calculation of the bending moment in a circular section can be determined by an integral equation. Considering only semi-membrane behaviour, the bending moment is as follows:

$M_{\mathrm{C}}=\int_{0}^{2 \pi} \sigma_{x} h r^{2} \cos \theta d \theta=\frac{E h}{1-\nu^{2}} \int_{0}^{2 \pi} \varepsilon_{x} r^{2} \cos \theta d \theta$

In a thin-walled element only the deformation along $x$ is considered for the calculation of the bending moment.

A similar calculation of the bending moment in the equivalent straight pipe is as follows:

$M_{\mathrm{S}}=\frac{E I}{\left(1-\nu^{2}\right)} \frac{\overline{\delta \alpha}}{L}$

The second moment of area for a thin tubular circular section is represented by:

$I=\pi r^{3} h$

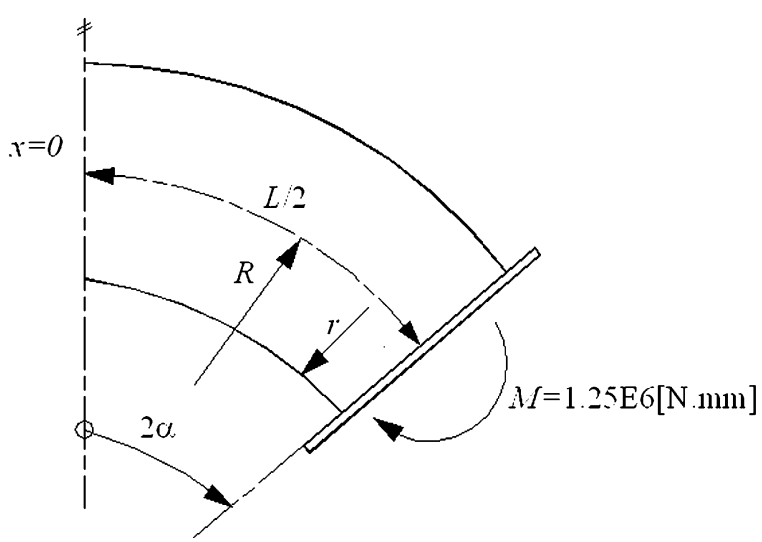

Fig. 2. Geometry of a curved pipe with end restraints. 
Table 1

Geometric and material properties for a curved pipe

\begin{tabular}{lllll}
\hline$D(\mathrm{~mm})$ & $h(\mathrm{~mm})$ & $R(\mathrm{~mm})$ & $L(\mathrm{~mm})$ & $E\left(\mathrm{~N} / \mathrm{mm}^{2}\right)$ \\
\hline 340 & 1.2 & 1110 & 826.5 & $7.36 \times 10^{4}$ \\
\hline
\end{tabular}

For the loading case of an imposed moment, the flexibility factors can be determined by the following equation

$K=\frac{\delta \alpha}{\overline{\delta \alpha}}$

where

$\overline{\delta \alpha}=M L\left(1-\nu^{2}\right) / E I$

and $\delta \alpha$ in a curved pipe is calculated by Eq. (16).

\section{The case study}

The studied case is shown in Fig. 2, representing a curved pipe with end restraints, subjected to a uniform bending moment. The geometry and the material properties are in accordance with Table 1, as in Ref. [7]. Only one half of the pipe bend was analysed due to geometric and loading symmetry. The results refer to the transverse section at $x=0$ in the case of rigid or thin flanges as shown in Fig. 2.

Figs. 3 and 4 show the longitudinal stresses of a curved pipe under pure bending moment, using rigid or thin flanges. Those results obtained with our formulation are compared with the results by Melo and Castro [7], and Wilczek [8]. We have calculated the longitudinal stresses using all terms or only odd terms in the Fourier expansions for the displacements calculation, represented along the semisection studied $\left(0-180^{\circ}\right)$.

When calculating the stress field for the curved pipes with rigid flanges we may use the formulation by all terms or only odd terms and will obtain the same results. But for the case studied with thin flanges, only the results obtained

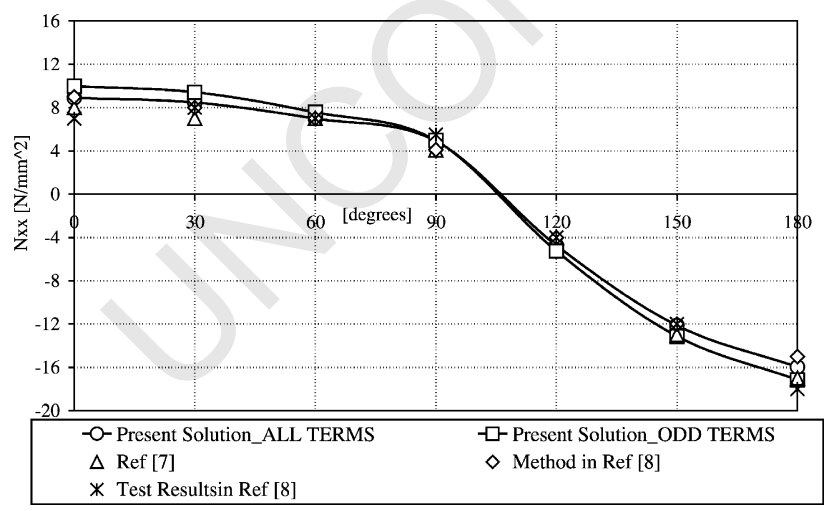

Fig. 3. Longitudinal stresses for a curved pipe with rigid flanges.

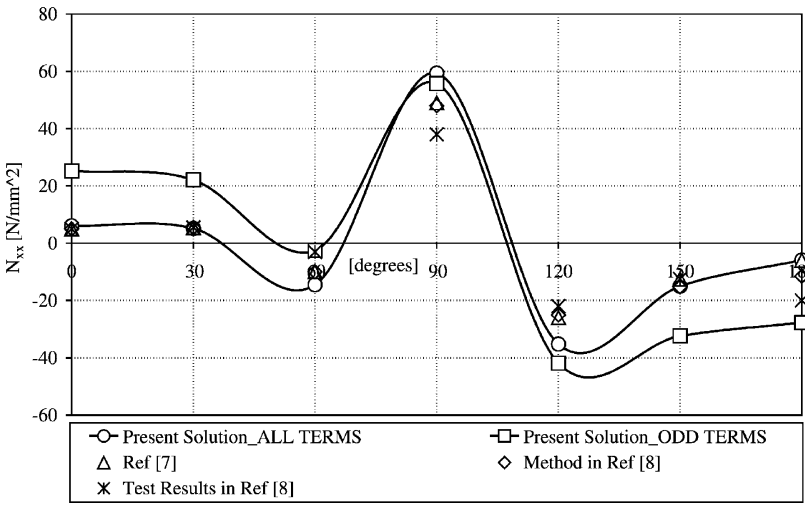

Fig. 4. Longitudinal stresses for a curved pipe with thin flanges.

with all terms are in good agreement with other references, as shown in Fig. 4.

\section{Stress analysis for other curved pipe geometry and end restraints}

In Table 2, 12 examples of $90^{\circ}$ elbows according ISO 1127 are presented. All cases refer to edge prescribed uniform bending moment. In the same way, only one half of the pipe bend was analysed and the results refer to the transverse section at $x=0$, as shown in Fig. 2.

In Figs. 5 and 6 we show the longitudinal and the meridional stresses for all curved pipes analysed. We have calculated the stresses in all pipes for this loading condition, having used either a complete formulation (all terms) or only odd terms in the Fourier expansions for the displacements evaluation. The top number of terms in the trigonometric expansions was fixed at eight. Next we present the results for a longitudinal membrane and meridional stress in curved pipe bends with end restraints using thick or thin flanges.

Table 2

Geometric parameters for $90^{\circ}$ elbows and material proprieties

\begin{tabular}{llrrr}
\hline$D(\mathrm{~mm})$ & $h(\mathrm{~mm})$ & $R(\mathrm{~mm})$ & $L(\mathrm{~mm})$ & $E\left(\mathrm{~N} / \mathrm{mm}^{2}\right)$ \\
\hline 21.30 & 2 & 31.95 & 50.19 & $2.10 \times 10^{5}$ \\
33.70 & 2 & 50.55 & 79.40 & $2.10 \times 10^{5}$ \\
60.30 & 2 & 90.45 & 142.08 & $2.10 \times 10^{5}$ \\
101.60 & 2 & 152.40 & 239.39 & $2.10 \times 10^{5}$ \\
323.90 & 2 & 485.85 & 763.17 & $2.10 \times 10^{5}$ \\
406.40 & 3 & 609.60 & 957.56 & $2.10 \times 10^{5}$ \\
508.00 & 3 & 762.00 & 1196.95 & $2.10 \times 10^{5}$ \\
609.60 & 3 & 914.40 & 1436.34 & $2.10 \times 10^{5}$ \\
711.20 & 4 & 1066.80 & 1675.73 & $2.10 \times 10^{5}$ \\
812.80 & 4 & 1219.20 & 1915.11 & $2.10 \times 10^{5}$ \\
914.40 & 4 & 1371.60 & 2154.50 & $2.10 \times 10^{5}$ \\
1016.00 & 4 & 1524.00 & 2393.89 & $2.10 \times 10^{5}$ \\
\hline
\end{tabular}


With rigid flanges
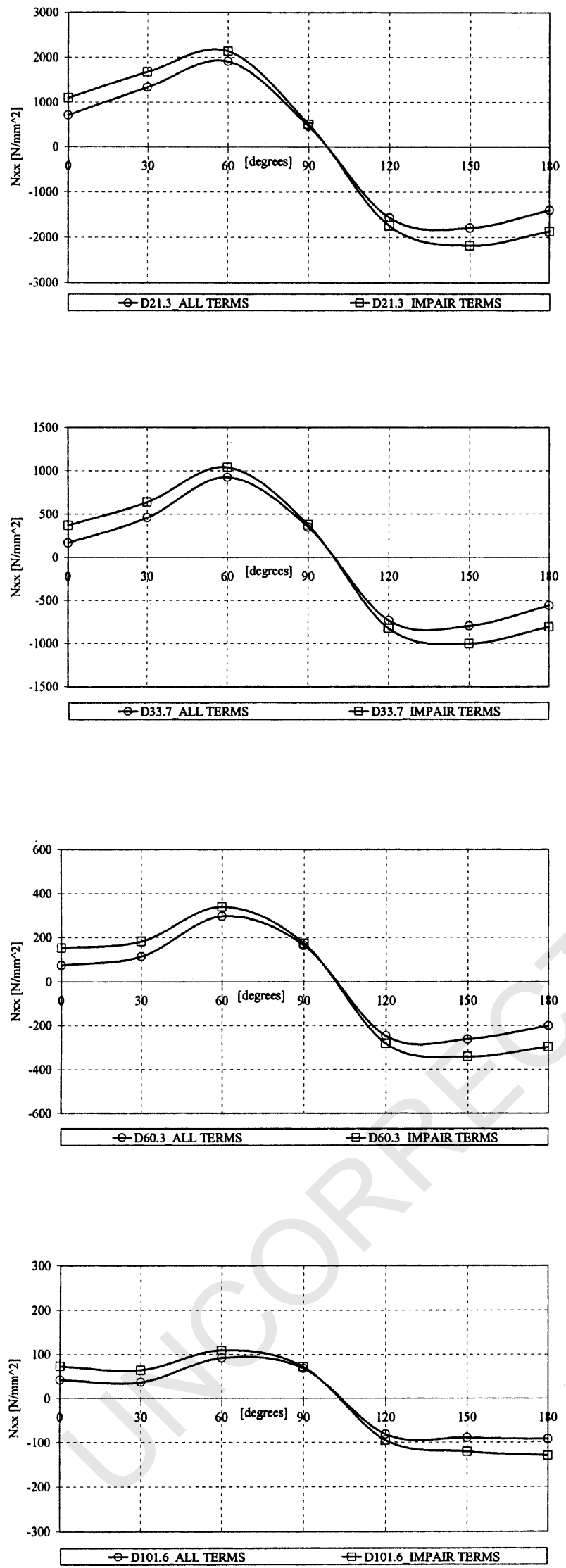

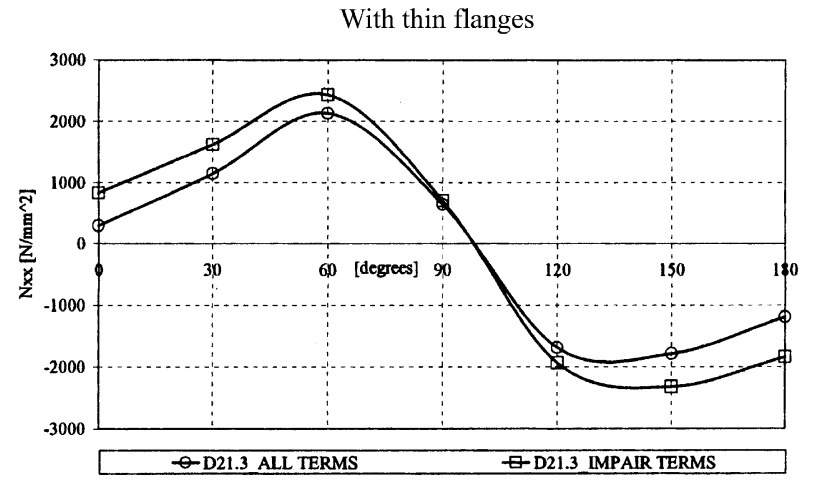

505

506

507

508

509

510

511

512

513

514

515

516

517

518

519

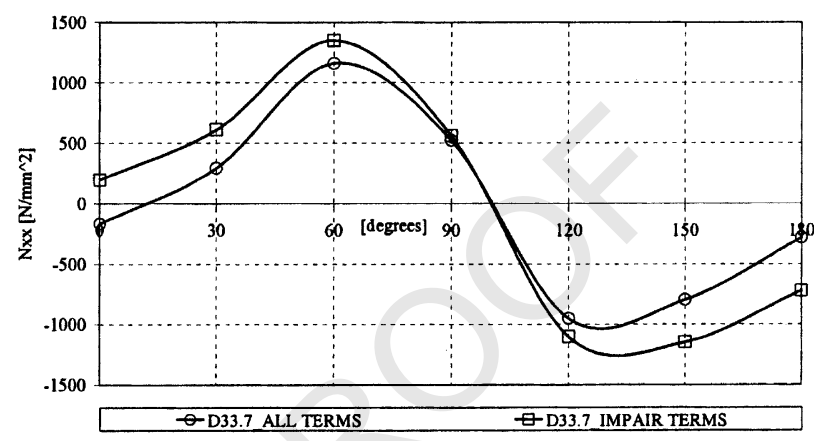

520

521

522

523

524

525

526

527

528

529

530

531

532

533

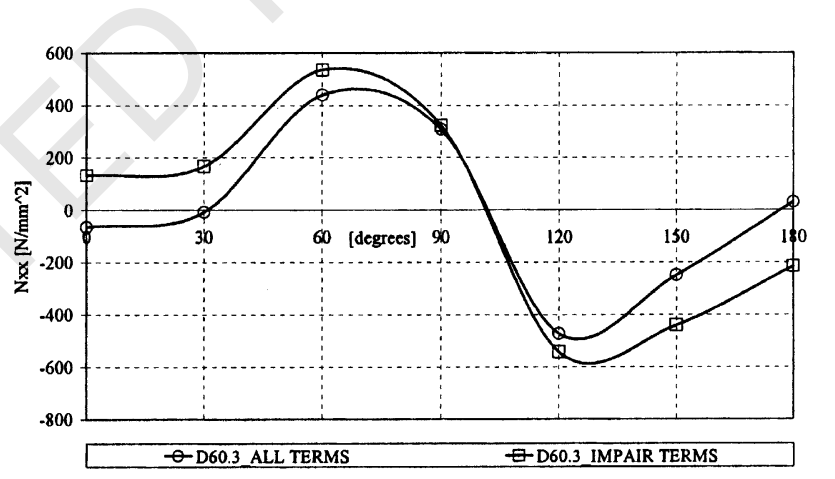

534

535

536

537

538

539

540

541

542

543

544

545

546

547

548

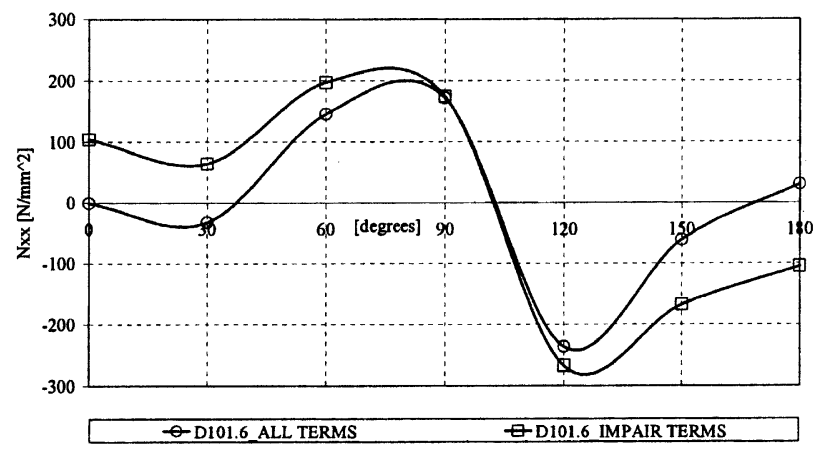

Fig. 5. Longitudinal stresses at section equidistant to edges $(x=0)$. 


\section{ARTICLE IN PRESS}

With rigid flanges
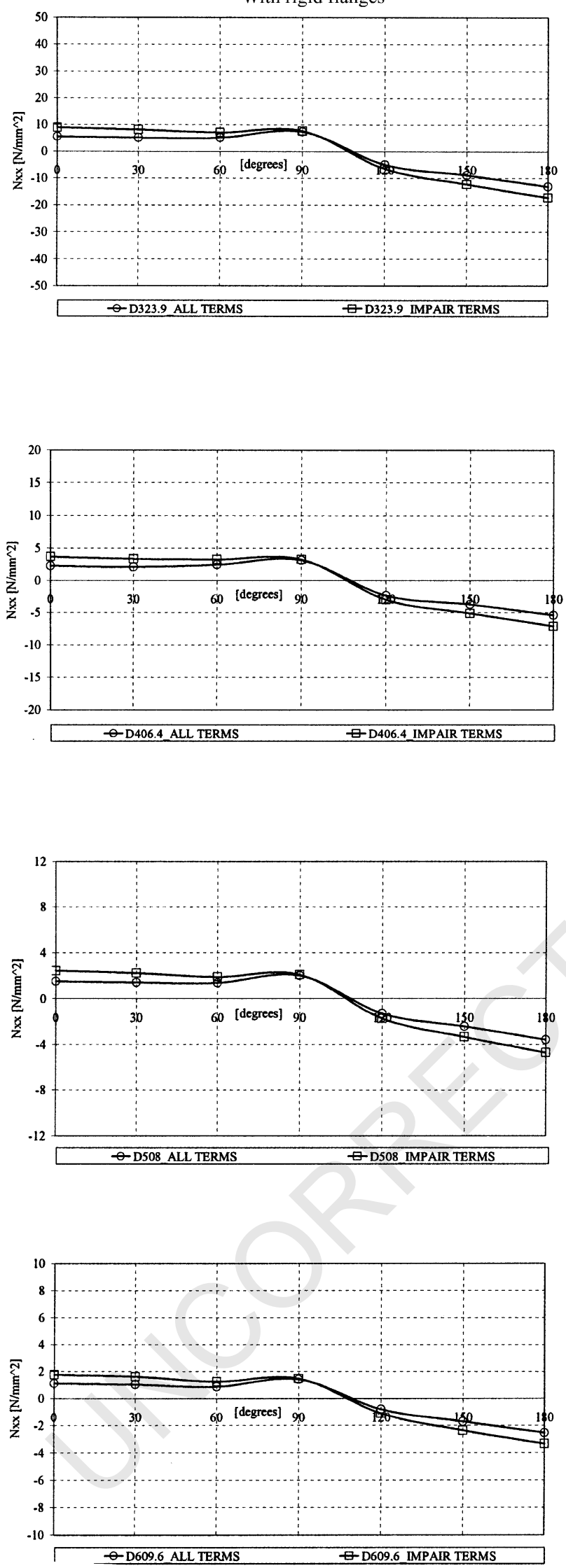

With thin flanges
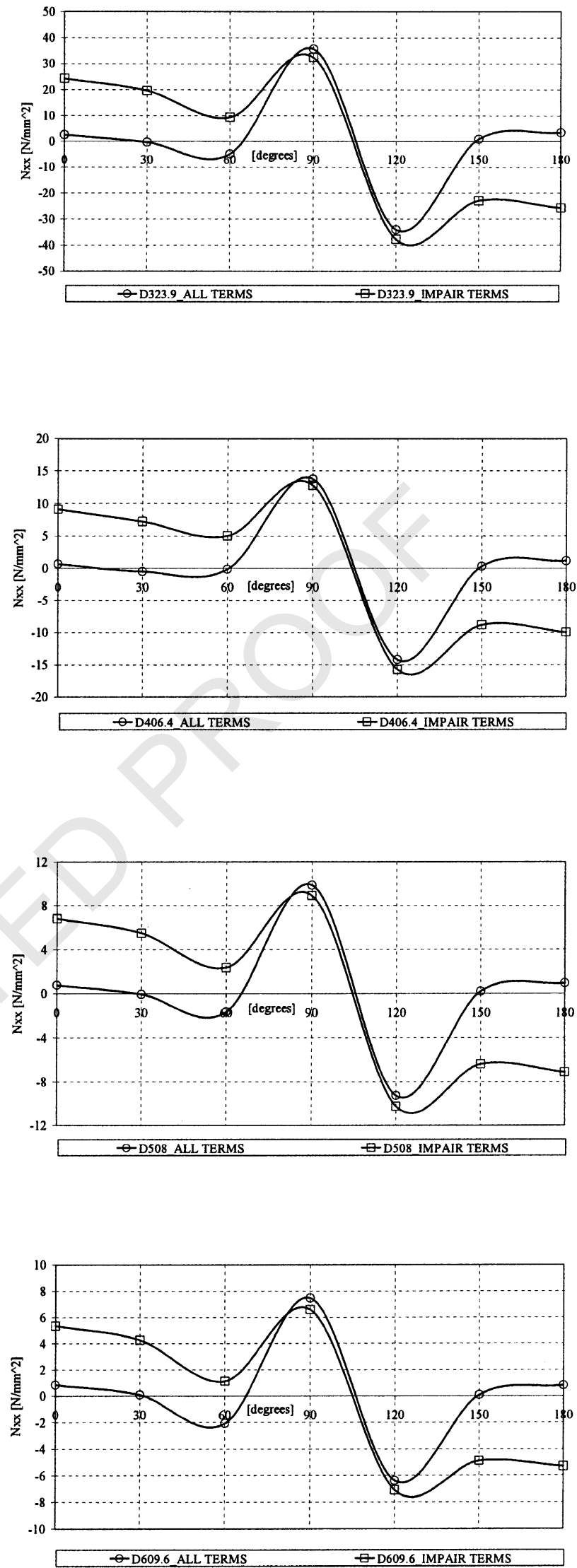

Fig. 5. (continued) 


\section{ARTICLE IN PRESS}

E.M.M. Fonseca et al. / International Journal of Pressure Vessels and Piping $x x(0000) x x x-x x x$

With rigid flanges

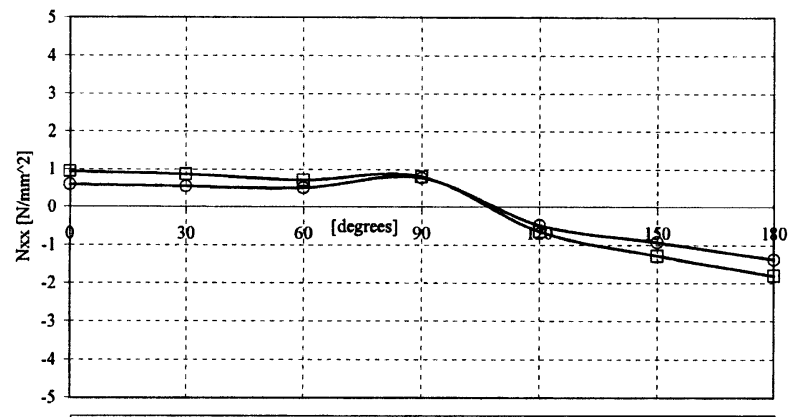

D711.2 ALL TRRMS

—D711.2 IMPAIR TERMS
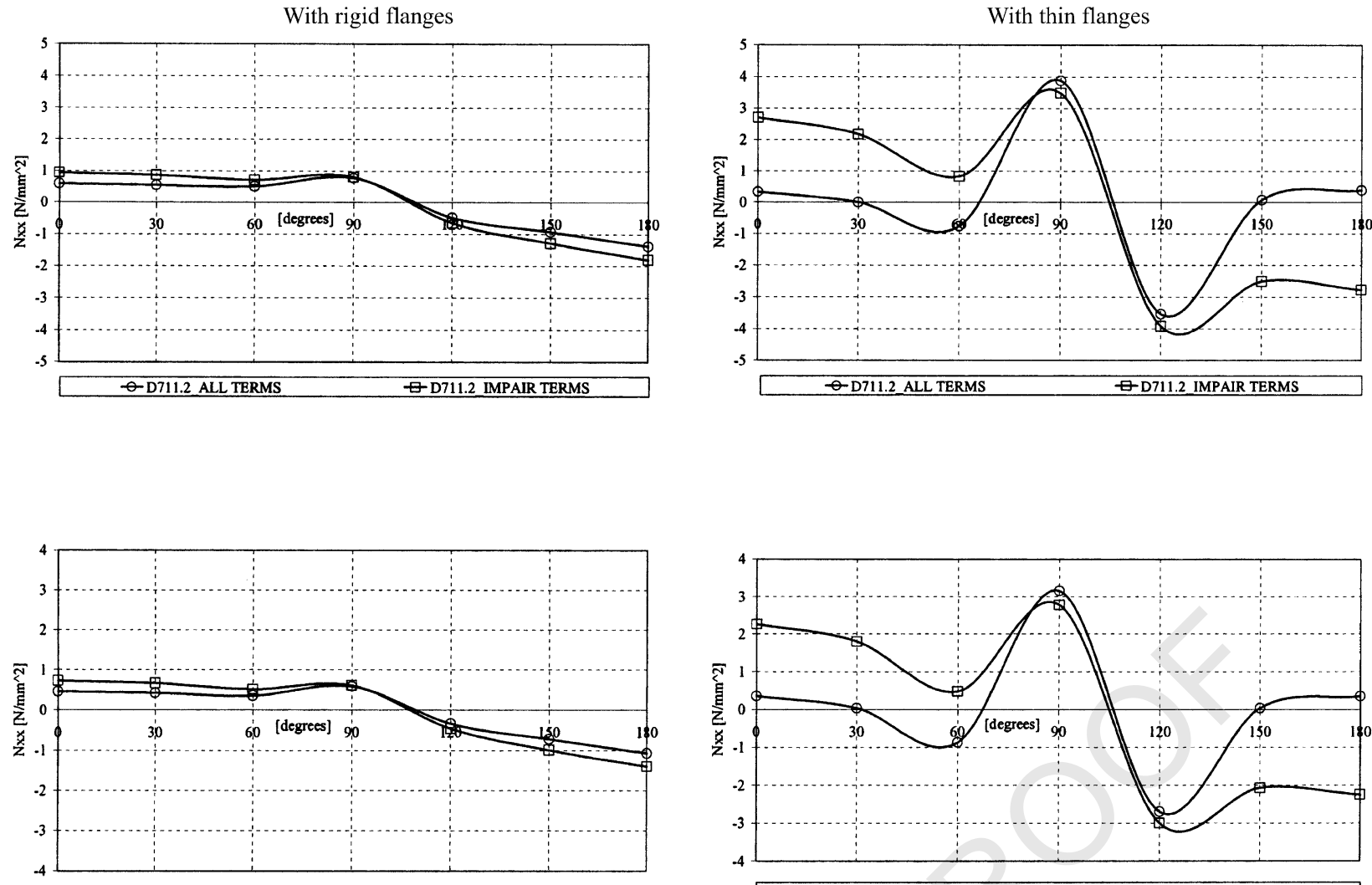

OD812.8 ALL TERMS
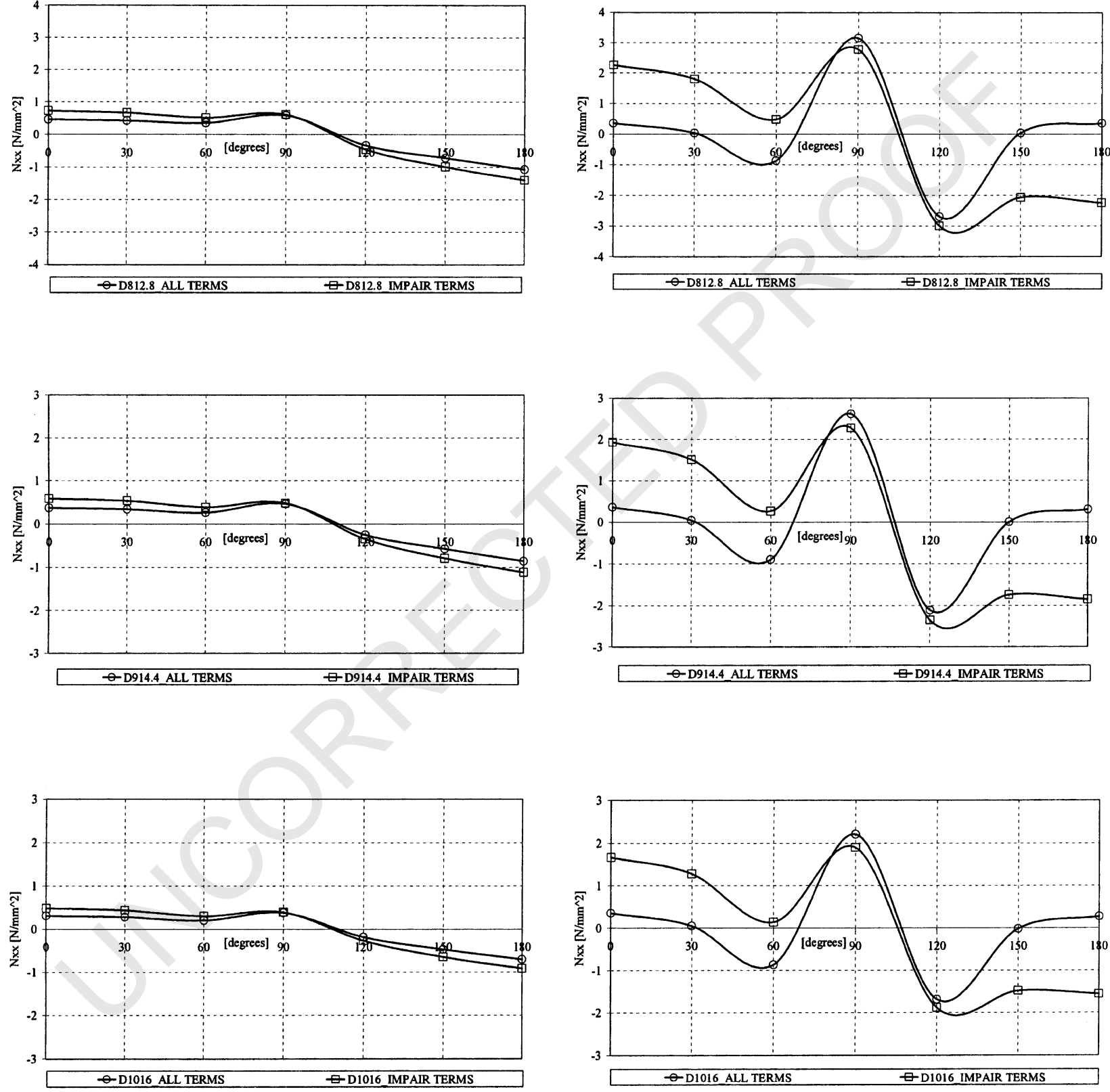

Fig. 5. (continued) 
With rigid flanges
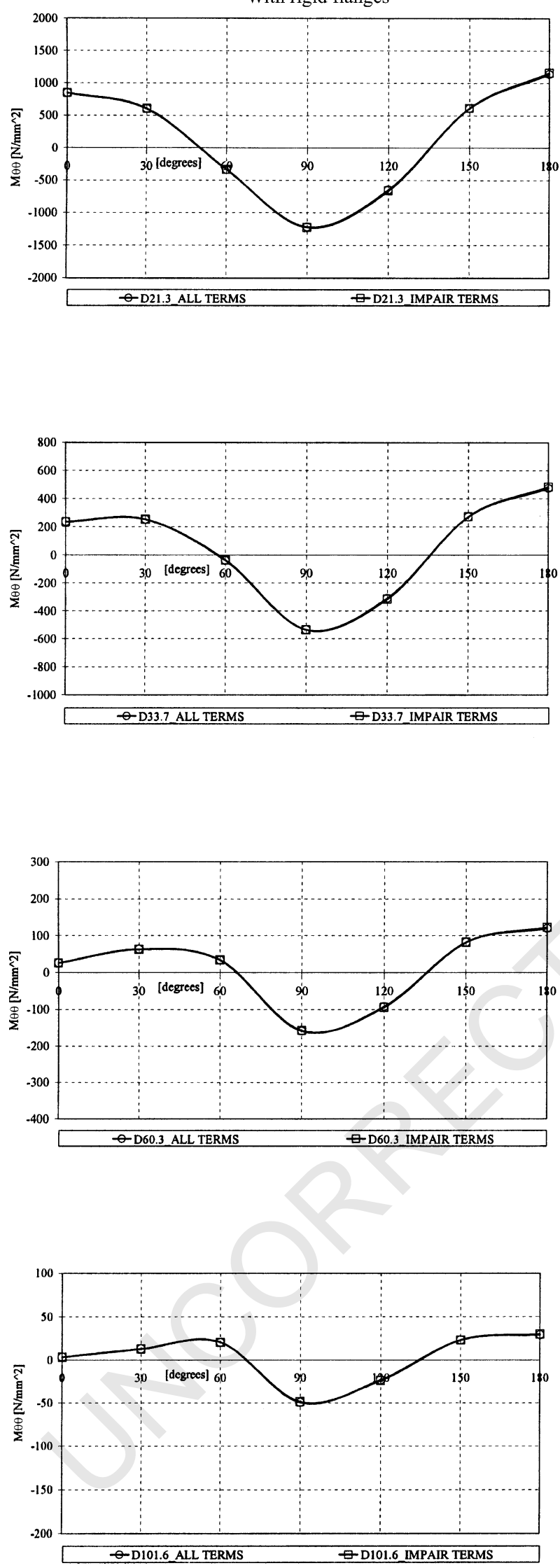

Fig. 6. Meridional bending stresses at section equidistant to edges (inside pipe surface).
With thin flanges
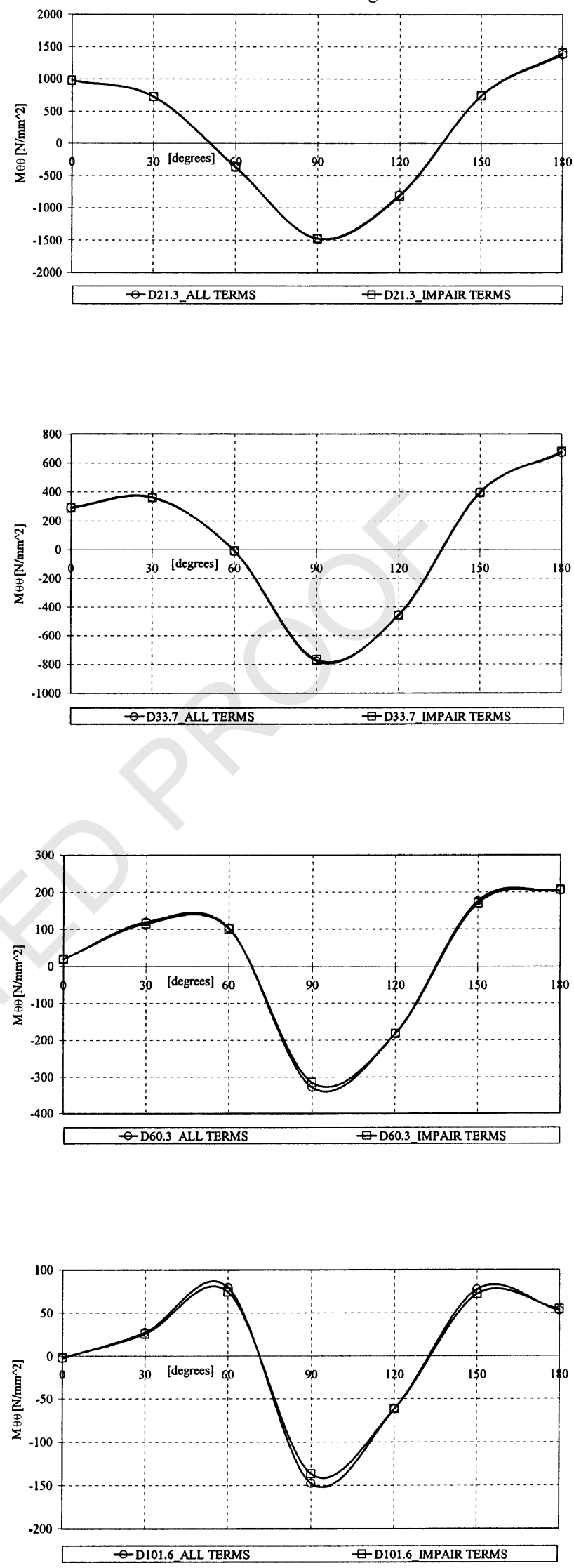


\section{ARTICLE IN PRESS}

E.M.M. Fonseca et al. / International Journal of Pressure Vessels and Piping xx (0000) xxx-xxx
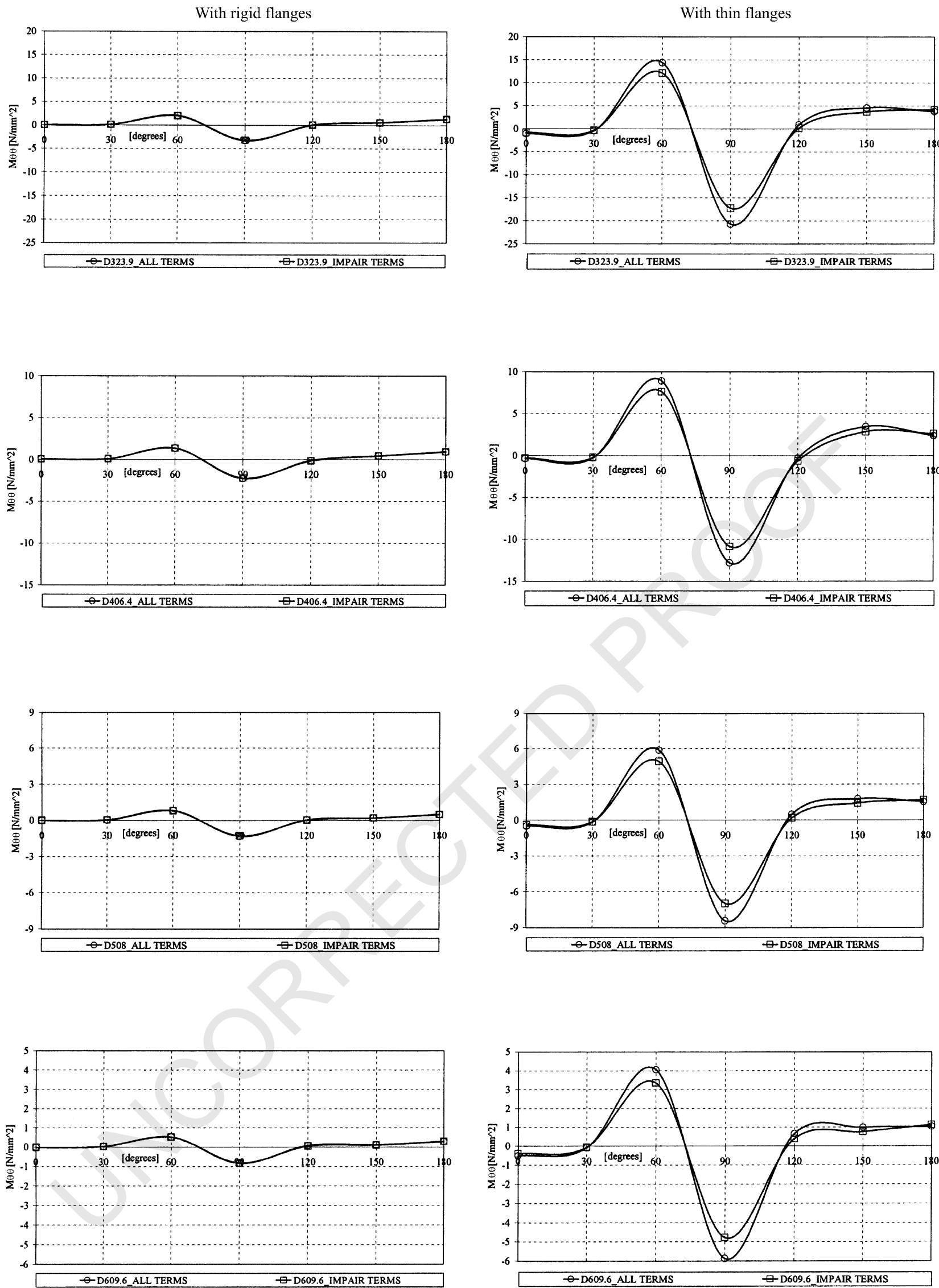

Fig. 6. (continued) 


\section{ARTICLE IN PRESS}

1009

1010

1011

1012

1013

1014

1015

1016

1017

1018

1019

1020

1021

1022

1023

1024

1025

1026

1027

1028

1029

1030

1031

1032

1033

1034

1035

1036

1037

1038

1039

1040

1041

1042

1043

1044

1045

1046

047

1052

1053

1054

1055

1056

1057

1058

1059

1060

1061

1062

1063

1064
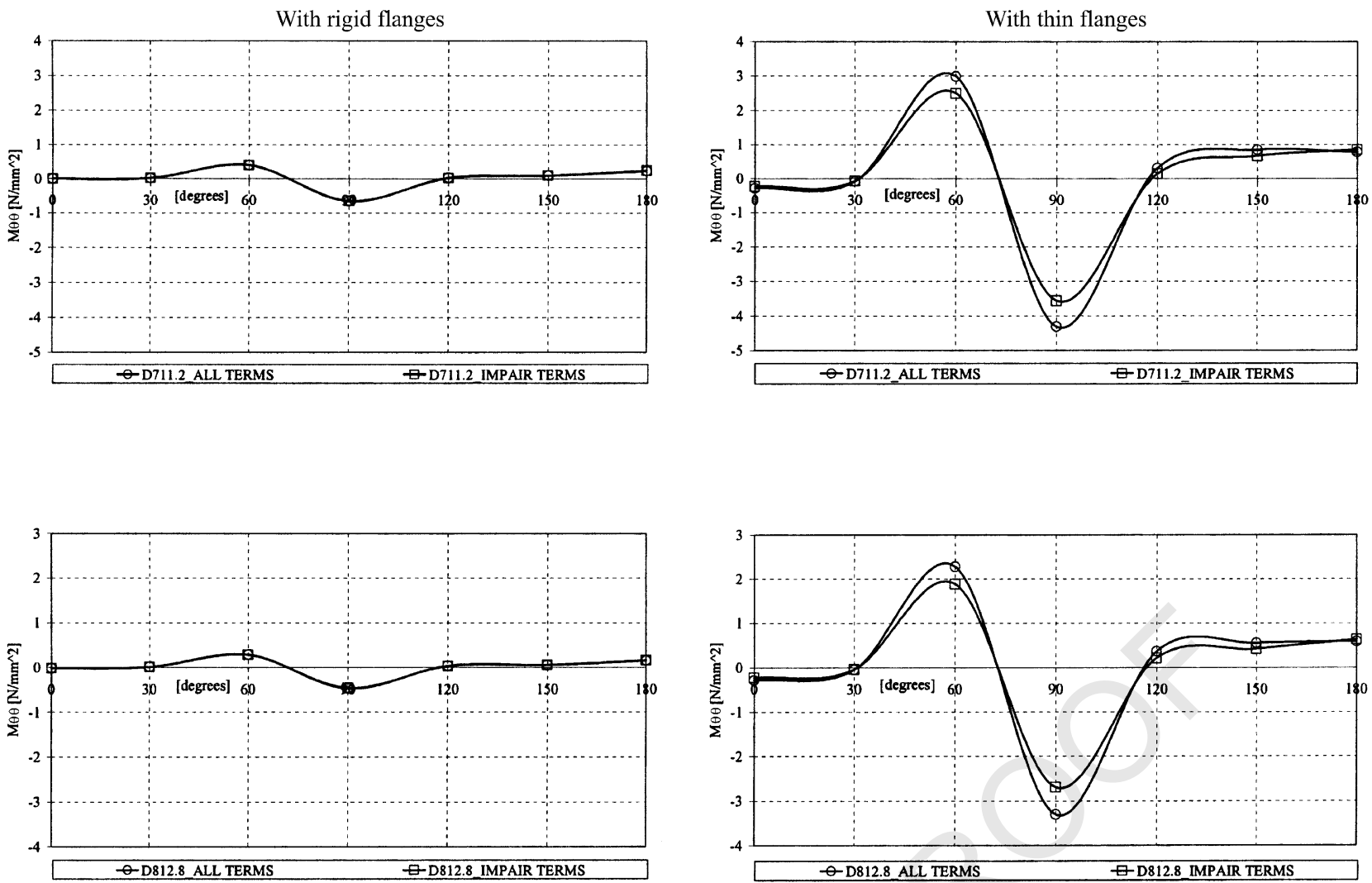

1065

1066

1067

1068

1069

1070

1071

1072

1073

1074

1075

1076

1077

1078

1079

1080

1081

1082

1083

1084

1085

1086

1087

1088

1089

1090

1091

1092

1093

1094

1095

1096

1097

1098

1099

1100

1101

1102

1103

1104

1105

1106

1107

1108

1109

1110

1111

1112

1113

1114

1115

1116

1117

1118

1119

1120 


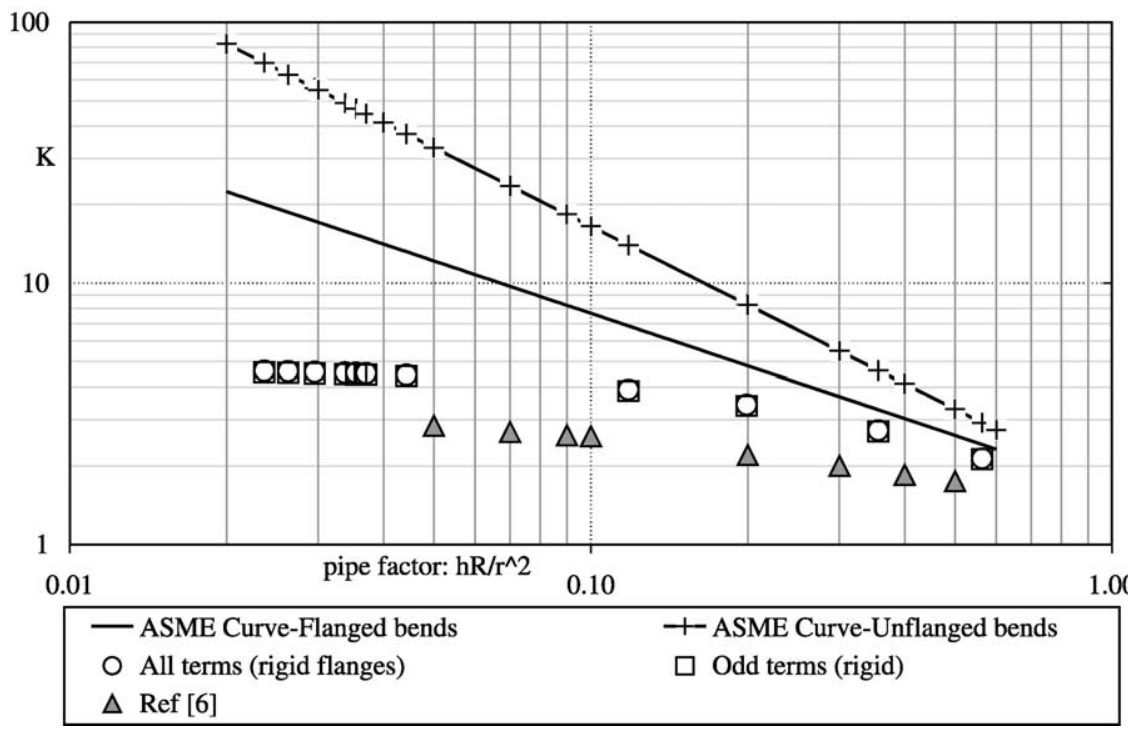

Fig. 7. Flexibility factors, curved pipes with rigid flanges.

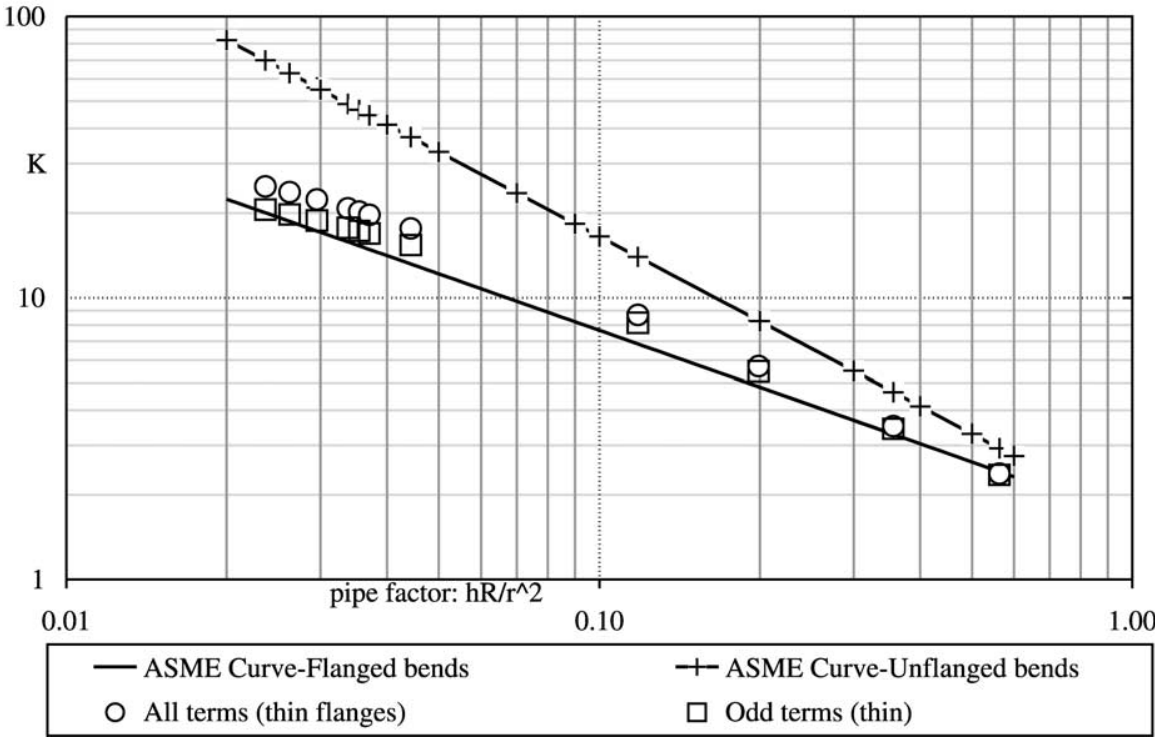

Fig. 8. Flexibility factors, curved pipes with thin flanges.

\section{Calculation of the flexibility factor}

According to the ASME Code, the calculation of the flexibility factor in a curved pipe under uniform bending is determined from the following equation:

$K=\frac{1.65}{\bar{h}^{2 / 3}} \quad$ (for flanged bends)

$K=\frac{1.65}{\bar{h}} \quad$ (for unflanged bends)

where

$\bar{h}=\frac{h R}{r^{2}}$
Fig. 7 shows the results obtained with the semi-analytic formulation discussed here compared with the ASME curve, considering rigid flanges for all curved pipes studied.

Fig. 8 shows the results obtained when using thin flanges. In this case the results obtained will be compared with the ASME curve for unflanged bends.

\section{Conclusion}

The present method is a procedure for the stress 1231 field determination or the displacement field calculations 


\section{References}

[1] Ohtaki S. FEM analysis of pipe bends subjected to out-of-plane bending. European Congress on Computational Methods in Applied Sciences and Engineering, ECCOMAS; 2000.

[2] Natarajan R, Mirza S. Effect of internal pressure on flexibility factors in pipe elbows with end constraints. J Pressure Vessel Technol 1985;107: 60-3.

[3] Madureira L, Melo FQ. A hybrid formulation in the stress analysis of curved pipes. Engng Comput 2000;17(8):970-80.

[4] Flugge W. Thin elastic shells. Berlin: Springer; 1973.

[5] Kitching R. Smooth and mitred pipe bends. In: Gill SS, editor. The stress analysis of pressure vessels and pressure. Oxford: Pergamon Press; 1970, chapter 7.

[6] Thomson G. In plane bending of smooth pipe bends. PhD Thesis. University of Strathclyde, Scotland, UK; 1980.

[7] Melo FJMQ, Castro PMST. A reduced integration Mindlin beam element for linear elastic stress analysis of curved pipes under generalized in-plane loading. Comput Struct 1992;43(4):787-94.

[8] Wilczek E. Statische Berechnung eines Rohrkrümmers mit Realen Randbedingungen. PhD Thesis. Technischen Hochschule Aachen, Aachen; 1984. 